\title{
Proton Therapy for Skull Base Chordomas: An Outcome Study from the University of Florida Proton Therapy Institute
}

\author{
Rohan L. Deraniyagala ${ }^{1}$ Daniel Yeung ${ }^{1} \quad$ William M. Mendenhall ${ }^{1}$ Zuofeng Li ${ }^{1} \quad$ Christopher G. Morris $^{1}$ \\ Nancy P. Mendenhall ${ }^{1}$ Paul Okunieff ${ }^{1}$ Robert S. Malyapa ${ }^{1}$
}

${ }^{1}$ University of Florida Proton Therapy Institute, Jacksonville, Florida, United States

Address for correspondence Robert S. Malyapa, MD, PhD, University of Florida Proton Therapy Institute, 2015 N Jefferson St, Jacksonville, FL

J Neurol Surg B 2014;75:53-57. 32206, United States (e-mail: rmalyapa@floridaproton.org).

\begin{abstract}
\section{Keywords}

- head and neck tumors

- chordoma

- outcomes

- proton therapy

- particle therapy

Objectives Skull base chordoma is a rare, locally aggressive tumor located adjacent to critical structures. Gross total resection is difficult to achieve, and proton therapy has the conformal advantage of delivering a high postoperative dose to the tumor bed. We present our experience using proton therapy to treat 33 patients with skull base chordomas.

Design Retrospective outcomes study.

Setting University of Florida Proton Therapy Institute; 2007 to 2011.

Participants A total of 33 patients with skull base chordomas received postoperative three-dimensional conformal proton therapy. The patients were $79 \%$ male and $6 \%$ diabetic; $27 \%$ had received a gross total resection.

Main Outcome Measures The gross tumor/tumor bed received a dose between 77.4 CGE and 79.4 CGE. Local control and overall survival were tracked, and radiation toxicity was assessed using a modified Radiation Therapy Oncology Group/European Organization for Research and Treatment of Cancer Late Radiation Morbidity Scoring Scheme. Results Median follow-up for all patients was 21 months. Local control and overall survival rates at 2 years were $86 \%$ and $92 \%$, respectively. Grade 2 toxicity was observed in $18 \%$ of our cohort in the form of unilateral hearing loss partially corrected with a hearing aid. No grade 2 or higher optic or brainstem toxicities were observed.

Conclusions Proton therapy is an effective treatment modality for skull base chordomas.
\end{abstract}

\section{Introduction}

Chordoma is a rare aggressive tumor that arises from the remnants of the notochord, which is the precursor to the nucleus pulposus or intervertebral disc. ${ }^{1}$ According to Surveillance Epidemiology and End Results data, the annual incidence is $\sim 0.08$ for 100,000 people. $^{2}$ Chordomas, although rarely metastatic, typically occur in a location adjacent to critical structures. The anatomical distribution of chordomas has been found to be $32.8 \%$ spinal, $32 \%$ cranial, and $29.2 \%$

received

March 14, 2013

accepted after revision

July 1, 2013

published online

September 9, 2013

sacral. ${ }^{3,4}$ Local progression of a base of skull chordoma can lead to severe neurologic deficits or death. The average time to death in an untreated skull base chordoma is between 6 and 24 months. ${ }^{5}$ Symptoms can be insidious, leading to a delay in diagnosis; Volpe et al showed that the median time from identification of symptoms to diagnosis was 14.6 months. ${ }^{6}$

The mainstay of treatment for a skull base chordoma is maximal tumor debulking. A gross total resection, however, is difficult to achieve, often leaving residual tumor. Local

C 2014 Georg Thieme Verlag KG Stuttgart · New York
DOI http://dx.doi.org/ 10.1055/s-0033-1354579. ISSN 2193-6331. 
recurrence with surgery alone has been found to be at least $58 \%$ and is associated with a significant postsurgical toxicity and mortality. ${ }^{7}$ Adjuvant radiotherapy (RT) is critical to achieve tumor control.

Adjuvant photon RT has been used extensively in the past. Historically, conventional opposed photon fields treating to a median dose of $60 \mathrm{~Gy}$ yielded local control rates from 23 to $39 \%$ and survival rates from 35 to $51 \%$ at 5 years. ${ }^{8,9}$ Debus et al used more conformal photon techniques and achieved a higher median dose to $66.6 \mathrm{~Gy}$, resulting in a 5-year local control rate of $50 \%{ }^{10}$ While suggesting a dose-response relationship, it appears that photon RT is limited by its conformality to achieve the necessary dose. Stereotactic radiosurgery (SRS) for skull base chordomas has shown improved or comparable local control for small tumors or recurrent disease. A study from Japan reported on 30 patients with skull base chordomas with a mean volume of $19.7 \mathrm{~cm}^{3}$ treated to a mean margin dose of $14 \mathrm{~Gy}$. The 5-year overall local control rate of $76 \%$ correlated only with a tumor volume of greater or less than $20 \mathrm{~cm}^{3}$ on univariate and multivariate analyses. ${ }^{11}$ Martin et al treated 18 chordoma patients with SRS to a mean marginal dose of 16.5 Gy and achieved a 5-year local control rate of $53 \%{ }^{12}$

Proton RT has an advantage over photon-based RT by using the Bragg peak to deliver a high conformal tumor dose to a target volume with very steep dose falloff, thus minimizing irradiation to adjacent critical structures. Physicians at the Massachusetts General Hospital (Boston, MA) have the most extensive experience using proton therapy for skull base chordomas. $^{13}$ Their 5- and 10-year local control rates were $73 \%$ and $54 \%$, respectively, using a tumor dose ranging from 66 to 83 CGE. We report our experience at the University of Florida Proton Therapy Institute (UFPTI) treating base of skull chordomas with protons.

\section{Methods and Materials}

A total of 33 patients with histologically confirmed skull base chordoma were enrolled from 2007 through 2011 in a prospective study approved by the institutional review board. Inclusion criteria were a primary site arising from the base of skull (sphenoid, clivus, petrous, or basiocciput), age 18 years or older at the time of consent, Karnofsky performance status $\geq 50$, neurologic function allowing the patient to cooperate with treatment, and surgery ranging from biopsy to gross total resection before RT. - Table 1 lists the patient and tumor characteristics for our study population.

Exclusion criteria included the following: prior RT to the head and neck, evidence of metastatic disease, serious medical or psychiatric illness that would preclude treatment compliance or informed consent, and pregnant or breastfeeding women. Only $27 \%$ of our patients had a gross total resection of the tumor.

For the simulation and treatment setup, each patient's head was supported on a carbon fiber Base of Skull (BoS) frame (Qfix, Avondale, PA) and immobilized with a customized thermal mask. The BoS frame is fabricated to ensure uniform attenuation of the proton beam over its entire
Table 1 Tumor and treatment characteristics

\begin{tabular}{|c|c|}
\hline Characteristic & Number (\%) of patients \\
\hline \multicolumn{2}{|c|}{ Presenting cranial nerve deficit } \\
\hline $\mathrm{VI}$ & $11(33)$ \\
\hline XII & $4(12)$ \\
\hline \multicolumn{2}{|c|}{ Brainstem involvement } \\
\hline Yes & $11(33)$ \\
\hline No & $22(67)$ \\
\hline \multicolumn{2}{|c|}{ Optic pathway involvement } \\
\hline Yes & $3(9)$ \\
\hline No & $30(91)$ \\
\hline \multicolumn{2}{|l|}{ Resection } \\
\hline GTR & $9(27)$ \\
\hline STR & $22(67)$ \\
\hline Biopsy Only & $2(6)$ \\
\hline
\end{tabular}

Abbreviations: GTR, gross total resection; STR, subtotal resection.

surface area. The design also allows maximum flexibility in field arrangements in conjunction with a robotic couch and a 360-degree proton gantry. Patient setup was conducted with care to ensure shoulder clearance and minimize the air gaps (and the lateral penumbra) of the treatment fields. Computed tomography (CT) images with and without intravenous contrast were acquired at $1-\mathrm{mm}$ spacing from the vertex to the base of skull. The CT image sets were registered with magnetic resonance imaging (MRI) data sets to guide the delineation of the target volumes and critical structures.

-Fig. 1 shows a patient with a clivus chordoma treated with proton therapy. The prescriptions were 50.4 CGE to the initial planning target volume (PTV 50.4) and 73.8 CGE to the boost volume (PTV 73.8). Because of the tendency of the target volume to wrap around the brainstem, and the target's close proximity to the visual apparatus, a patch-field technique unique to proton treatment is often used as part of the field arrangement. In the example shown, the initial target volume, PTV 50.4, was treated with five fields including a pair of patch fields. In the first pair, the left anterior oblique through field (LAO-T) was intentionally blocked to avoid the brainstem, missing the posterior aspect of the target as a result. The residual target was treated, or "patched," with a left posterior oblique patch field (LPO-P), which was feasible because of the finite range characteristics of the proton beam. The $50 \%$ dose level of the distal falloff of the LPO-P was adjusted to match the $50 \%$ dose corresponding to the field edge of the LAO-T beam. Ideally, the dose gradient of the distal falloff should closely match that of the lateral penumbra to achieve a homogeneous dose at the junction and within the target volume. In reality, there could be hot and cold spots near the junction. The second pair, composed of the right anterior oblique (RAO-T) through beam and the right posterior oblique patch (RPO-P) beam, somewhat mirrored the first so as to minimize the uncertainties and the impact of the 

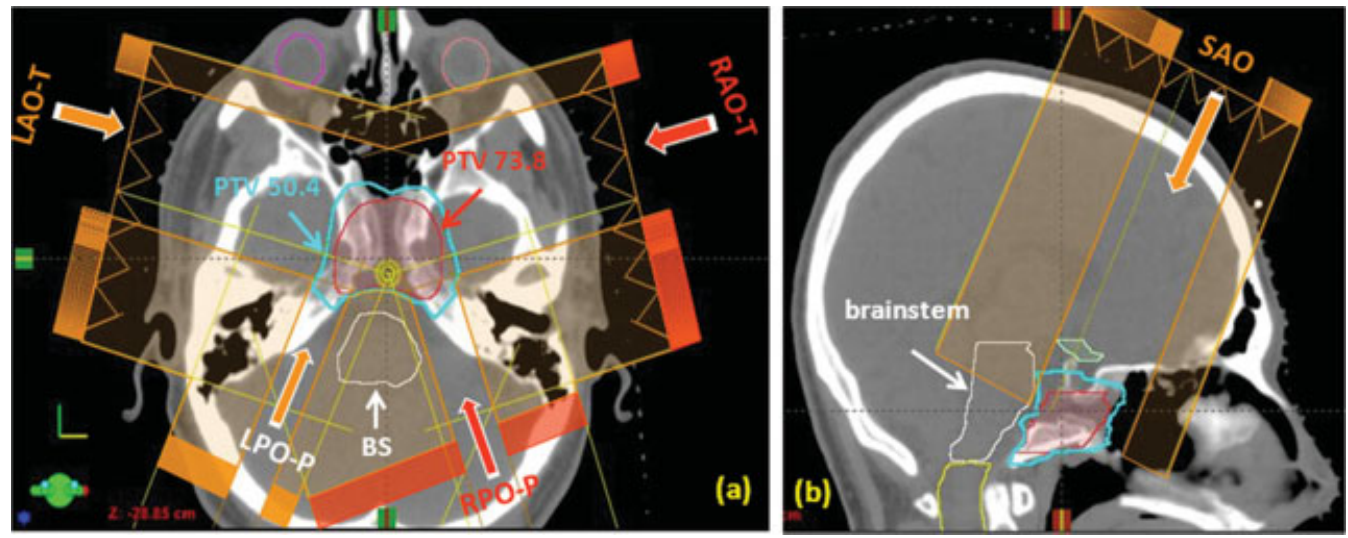

Fig. 1 A patient with a clivus chordoma. Five treatment fields were used for the planning target volume (PTV) 50.4 CGE (delivered dose: 54 CGE). (A) Axial view showing the two pairs of patch fields: First pair is left anterior oblique-through (LAO-T) and left posterior oblique-patch (LPO-P); second pair is right anterior oblique-through (RAO-T) and right posterior oblique-patch (RPO-P). The through beams (LAO-T and RAO-T) are laterally blocked to avoid the brainstem. The residual target volumes are covered by the patch fields (LPO-P and RPO-P) coming from the posterior. (B) Sagittal view showing the 5th superior anterior oblique (SAO) beam. This beam angle allows the brainstem to be blocked while essentially covering the whole target. Also shown is the boost target volume, PTV 73.8 CGE.

hot and cold spots that could arise at the junctions. Note that both the RAO and LAO fields were selected so that the chiasm and optic nerves could be blocked. To further improve the target coverage, a superior anterior oblique (SAO) field was added with an angle that allowed the brainstem to be

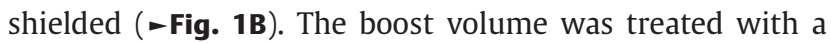
similar field arrangement.

- Fig. 2 shows the isodose in color wash and the dosevolume histogram of the composite plan. The dose plan was designed with high priority to limit the dose to the critical structures. The $0.1 \mathrm{~cm}^{3}$ doses to the brainstem, spinal cord, chiasm, and optic nerves as well as the expanded volume
(5 $\mathrm{mm}$ for spinal cord and $3 \mathrm{~mm}$ for brainstem, chiasm, and optic nerves) are tabulated in - Table 2. The dose levels achieved were well under the established dose tolerances for these structures. Despite our conservative approach, adequate doses for the target volumes were also achieved. Nearly $100 \%$ of the initial target volume received the treatment dose of 50.4 CGE; $90 \%$ of the boost volume received the treatment dose of 73.8 CGE. To minimize the risk of necrosis, the hot spot within the target ( $2 \%$ volume) was limited to $114 \%$ of the prescription dose. Daily treatments were delivered with a digital online image guidance system, and a setup accuracy $>2$ mm was achieved.
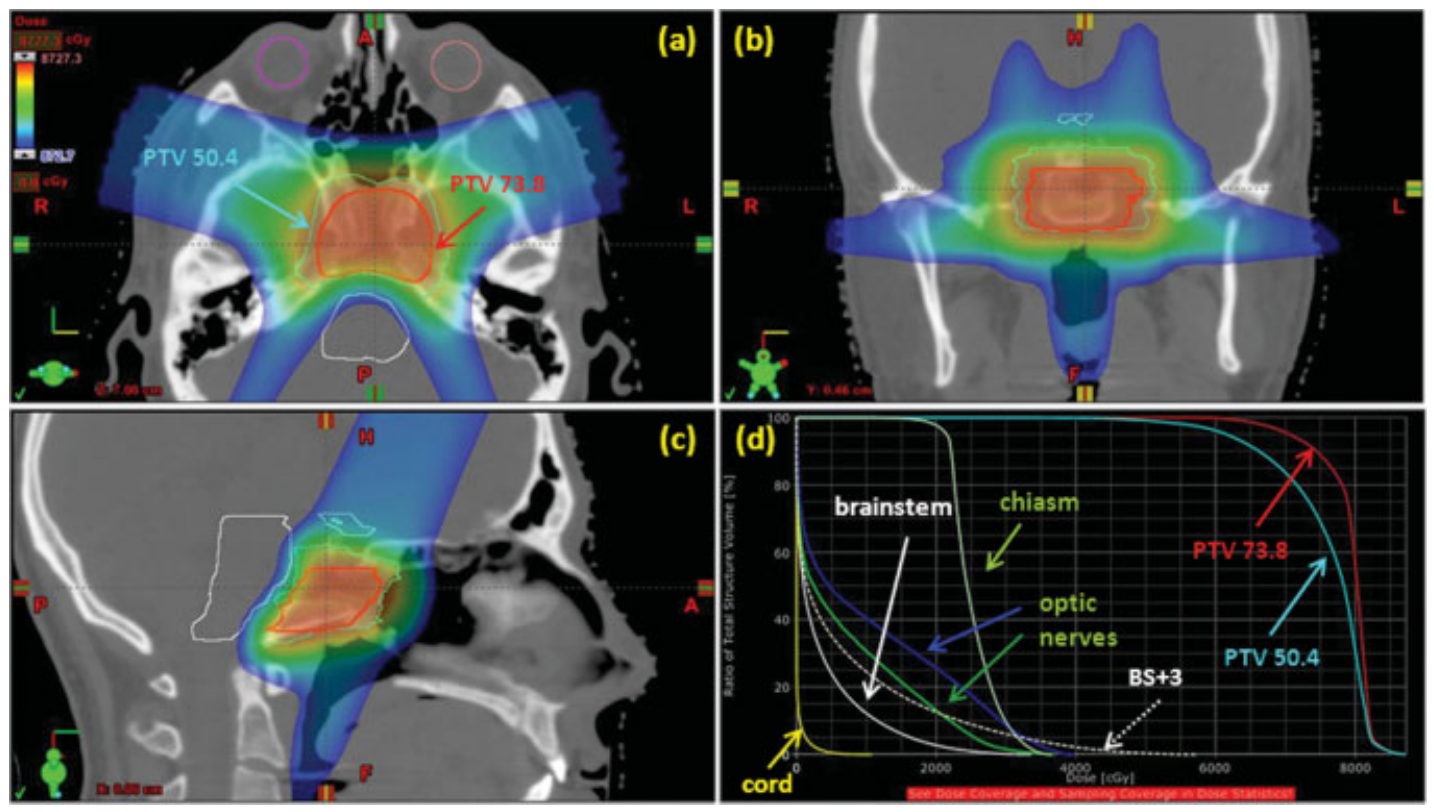

Fig. 2 (A-C). Isodose shown in color wash for the composite plan. The prescription dose to the planning target volume (PTV) was 50.4 CGE for the initial target volume and 73.8 CGE for the boost volume. (D) Dose-volume histogram distribution for the composite plan. Note: BS $+3=$ brainstem plus 3-mm expansion. 
Table 2 Dose constraint results

\begin{tabular}{|l|l|}
\hline Constraint & $\begin{array}{l}\text { Number (\%) of patients } \\
\text { who met the goal }\end{array}$ \\
\hline PTV D95 $=100 \%$ & $29(88)$ \\
\hline $\begin{array}{l}\text { Brainstem (center) } \\
0.1 \mathrm{~cm}^{3}<55 \text { CGE }\end{array}$ & $29(88)$ \\
\hline Cord $0.1 \mathrm{~cm}^{3}<50$ CGE & $33(100)$ \\
\hline Chiasm $0.1 \mathrm{~cm}^{3}<55$ CGE & $31(94)$ \\
\hline $\begin{array}{l}\text { Right optic nerve } \\
0.1 \mathrm{~cm}^{3}<55 \text { CGE }\end{array}$ & $32(97)$ \\
\hline $\begin{array}{l}\text { Left optic nerve } \\
0.1 \mathrm{~cm}^{3}<55 \text { CGE }\end{array}$ & $33(100)$ \\
\hline
\end{tabular}

Abbreviation: PTV, planning target volume.

Patients received a median PTV dose of 74 CGE (range: 7079 CGE). Patients were followed for a median of 21 months (range: 3-58 months). Skull base MRs were obtained every 6 months.

Toxicities were scored using a modified Radiation Therapy Oncology Group/European Organization for the Research and Treatment of Cancer Late Radiation Morbidity Scoring Scheme.

All statistical computations were accomplished with SAS and JMP software (SAS Institute, Cary, NC). The Kaplan-Meier product limit provided estimates of local control and overall survival. ${ }^{14}$

\section{Results}

With regard to surgical toxicities, 10 patients experienced a cerebrospinal fluid leak, 4 patients had postoperative meningitis, and 4 patients experienced a new cranial nerve deficit after resection.

The local control and overall survival rates at 2 years were $86 \%$ and $92 \%$, respectively (-Fig. 3). One patient progressed through treatment and was put on salvage immunomodulator therapy. Three patients (9\%) had an in-field recurrence after treatment. No patient experienced a marginal recur-

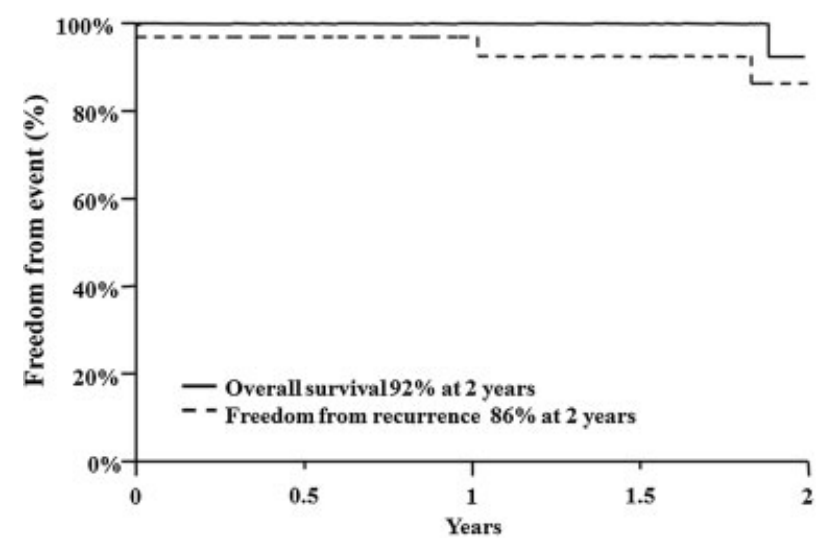

Fig. 3 Kaplan-Meier curves for overall survival and freedom from recurrence rates at 2 years. rence or distant metastases. One patient died from disease progression.

The only radiation-related grade 2 or higher toxicity was unilateral hearing loss partially corrected by a hearing aid, which was observed in $18 \%$ of our patients. Endocrine toxicity data were largely unavailable to us because many of our patients were located in different states or internationally. There were no grade 2 or higher brainstem or visual toxicities.

\section{Discussion}

Skull base chordomas are locally aggressive and infiltrative tumors surrounded by critical structures and often cause debilitation or death. ${ }^{15}$ Surgery is the primary treatment, but the high likelihood of residual tumor requires optimal adjuvant RT. Our outcomes when treating this disease with adjuvant proton therapy are similar to those reported by investigations at other proton therapy institutions.

Maximal debulking is critical to control of a skull base chordoma, but, as seen in our experience, gross total resection is difficult to achieve. ${ }^{16}$ At the University of Arkansas, gross total resection was achieved in 10 of 25 patients (40\%). ${ }^{17}$ Gay at el reported a $47 \%$ rate of near-total resection among 60 patients with skull base chordomas and chondrosarcomas. ${ }^{18}$ Although most of our patients underwent multiple operations, our gross total resection rate was $27 \%$. Thus adjuvant proton therapy was predominantly delivered in the setting of gross residual disease. Furthermore, 30\% of our patients experienced a cerebrospinal fluid leak, and $12 \%$ had postoperative meningitis following surgery. These surgical toxicities are similar to other reported series. ${ }^{17,18}$

As with other studies, ${ }^{13,19,20}$ our volume definition was based on preoperative and postoperative MRI and CT images. Our normal-tissue constraints were also similar, as shown in - Table 2. We saw only in-field recurrences and achieved a local control rate of $86 \%$ at 2 years, which makes our findings comparable with the published data. Hug et al reported a series of 33 patients with chordoma treated at Loma Linda University to a median dose of 70.7 CGE with a 5-year local control of $59 \% .{ }^{19}$ A series from Massachusetts General Hospital in which 621 patients were treated with a mixture of photons and protons to between 66 and 83 CGE showed 5and 10 -year local control rates of $73 \%$ and $54 \%$, respectively. ${ }^{13}$ Ares et al reported on their experience using the spotscanning technique of proton delivery to treat 42 patients with skull base chordomas and observed a 5-year local control rate of $81 \%$ with this highly conformal technique. ${ }^{20}$

Because the main site of recurrence is local and the salvage of local recurrences is unlikely, particularly in previously resected patients, survival is directly correlated with local control. Other institutions have reported 5-year survival rates of $59 \%, 73 \%$, and $81 \% .^{13,19,20}$

At 2 years, our patients have not experienced any brainstem or optic toxicities, and other series have shown low toxicity rates as well. Debus et al reported a 5.5\% rate of brainstem symptoms in 19 of 348 evaluable patients. The median time to symptoms was 17 months. On multivariate analysis, there was a statistically significant increase in the 
rate of brainstem symptoms when the volume of brainstem receiving 60 CGE was $>0.9 \mathrm{~cm}^{3} .{ }^{21,22}$ Of our patients, $88 \%$ of patients received $<55 \mathrm{CGE}$ to $0.1 \mathrm{~cm}^{3}$ of the brainstem. Pai et al reported on endocrine toxicity for 107 patients with skull base chordomas and chondrosarcomas. The 5- and 10-year rates for hyperprolactinemia were $72 \%$ and $84 \%$ and for hypothyroidism were $30 \%$ and $63 \% .^{3}$ These endocrinopathies were observed when the minimum dose to the pituitary exceeded 50 CGE $D_{\min }>50$. At present, we do not have the endocrine laboratory results for our patients.

\section{Conclusions}

Our experience treating patients with skull base chordomas with proton therapy is promising in terms of control of this locally aggressive tumor. In view of the high dose delivered and the proximity of adjacent critical structures, the toxicity profile is acceptable. With longer follow-up, we expect our 5and 10-year local control and survival rates to approach those reported by other investigators.

\section{Acknowledgments}

We would like to thank Karen Lopez-Bunk, RN, for her care of the patients included in this study.

\section{References}

1 Weber AL, Liebsch NJ, Sanchez R, Sweriduk ST Jr. Chordomas of the skull base. Radiologic and clinical evaluation. Neuroimaging Clin $\mathrm{N}$ Am 1994;4(3):515-527

2 McMaster ML, Goldstein AM, Bromley CM, Ishibe N, Parry DM. Chordoma: incidence and survival patterns in the United States, 1973-1995. Cancer Causes Control 2001;12(1):1-11

3 Pai HH, Thornton A, Katznelson L, et al. Hypothalamic/pituitary function following high-dose conformal radiotherapy to the base of skull: demonstration of a dose-effect relationship using dosevolume histogram analysis. Int J Radiat Oncol Biol Phys 2001; 49(4):1079-1092

4 Mendenhall WM, Mendenhall CM, Lewis SB, Villaret DB, Mendenhall NP. Skull base chordoma. Head Neck 2005;27(2):159-165

5 Kamrin RP, Potanos JN, Pool JL. An evaluation of the diagnosis and treatment of chordoma. J Neurol Neurosurg Psychiatry 1964;27: 157-165
6 Volpe NJ, Liebsch NJ, Munzenrider JE, Lessell S. Neuro-ophthalmologic findings in chordoma and chondrosarcoma of the skull base. Am J Ophthalmol 1993;115(1):97-104

7 Pamir MN, Kiliç T, Türe U, Ozek MM. Multimodality management of 26 skull-base chordomas with 4-year mean follow-up: experience at a single institution. Acta Neurochir (Wien) 2004; 146(4):343-354

8 Zorlu F, Gürkaynak M, Yildiz F, Oge K, Atahan IL. Conventional external radiotherapy in the management of clivus chordomas with overt residual disease. Neurol Sci 2000;21(4):203-207

9 Forsyth PA, Cascino TL, Shaw EG, et al. Intracranial chordomas: a clinicopathological and prognostic study of 51 cases. J Neurosurg 1993;78(5):741-747

10 Debus J, Schulz-Ertner D, Schad L, et al. Stereotactic fractionated radiotherapy for chordomas and chondrosarcomas of the skull base. Int J Radiat Oncol Biol Phys 2000;47(3):591-596

11 Hasegawa T, Ishii D, Kida Y, Yoshimoto M, Koike J, Iizuka H. Gamma knife surgery for skull base chordomas and chondrosarcomas. J Neurosurg 2007;107(4):752-757

12 Martin JJ, Niranjan A, Kondziolka D, Flickinger JC, Lozanne KA, Lunsford LD. Radiosurgery for chordomas and chondrosarcomas of the skull base. J Neurosurg 2007;107(4):758-764

13 Munzenrider JE, Liebsch NJ. Proton therapy for tumors of the skull base. Strahlenther Onkol 1999;175(Suppl 2):57-63

14 Kaplan EL, Meier P. Nonparametric estimation from incomplete observations. J Am Stat Assoc 1958;53:457-481

15 Rich TA, Schiller A, Suit HD, Mankin HJ. Clinical and pathologic review of 48 cases of chordoma. Cancer 1985;56(1):182-187

16 Tai PT, Craighead P, Bagdon F. Optimization of radiotherapy for patients with cranial chordoma. A review of dose-response ratios for photon techniques. Cancer 1995;75(3):749-756

17 al-Mefty O, Borba LA. Skull base chordomas: a management challenge. J Neurosurg 1997;86(2):182-189

18 Gay E, Sekhar LN, Rubinstein E, et al. Chordomas and chondrosarcomas of the cranial base: results and follow-up of 60 patients. Neurosurgery 1995;36(5):887-896; discussion 896-897

19 Hug EB, Loredo LN, Slater JD, et al. Proton radiation therapy for chordomas and chondrosarcomas of the skull base. J Neurosurg 1999;91(3):432-439

20 Ares C, Hug EB, Lomax AJ, et al. Effectiveness and safety of spot scanning proton radiation therapy for chordomas and chondrosarcomas of the skull base: first long-term report. Int J Radiat Oncol Biol Phys 2009;75(4):1111-1118

21 Debus J, Hug EB, Liebsch NJ, et al. Brainstem tolerance to conformal radiotherapy of skull base tumors. Int J Radiat Oncol Biol Phys 1997;39(5):967-975

22 Devisetty K, Malyapa RS, Mendenhall WM. Tumors of the base of skull. In: Mehta M, ed. Principles and Practice of Neuro-Oncology: A Multidisciplinary Approach. New York, NY: Demos Medical Publishing; 2011:846-860 